DISCUSSION.

Dr. W. W. Grant of Denver-The unequivocal statement of the essavist that all these cases can be treated by wiring the fragments demands consideration. While it is a fact that Lord Lister established the aseptic treatment of wiring the patella on a sound and enduring basis, I will say that l went through the wards with him in London eight or ten years ago and observed his cases, which he took great pains to explain in detail, and his practice was to use large silver wire. I never saw a case of suppuration, and always a good result. The probabilities are that he would not have the same results with the same treatment today. Yet it is a fact that the profession is not now agreed that it is wise to make a compound fracture of a simple fracture of the patella in order to wire it. As a matter of fact, the result of close ligamentous union is not bad; if the patient has not that bony substantial union that he would have by successful wiring. It must be remembered that, in spite of antiseptic precautions, infection sometimes gets in to these wounds, and if such should be the case, there will not only be imperfect union but an ankylosed joint, and surgeons hesitate very much to convert simple fractures of the patella into compound ones. I do not believe such a recommendation would be safe for general adoption. It must be admitted, too, that if the case should fall into the hands of a skilled surgeon, who is always careful to carry out every aseptic precaution, the result will be gratifying, but this will often not be the case, and if it is once generally known as the ultimatum of the profession, that in cases of considerable effusion of blood into the joint the joint should be opened, the clots of débris turned out, and the fragments wired, we are going to have some bad results follow which will bring the operation into disrepute. There is no question but that in compound fracture of the patella, the patella should be wired at once. In those cases where we have considerable laceration of the soft parts, extreme distension, with effusion of blood, it is wise to open and turn out the blood.clots; but in cases seen around the elbow-joint these can be dealt with very well by aspiration. At the pres. ent time it is hardly a safe dictum that we should convert simple fractures of the patella into compound ones for the purpose of wiring the fragments together, in order to get bony union, while under our present methods of treatment we can secure close ligamentous union with very good results.

Dr. FreD J. Hodaes of Ashland, Wis. - The essayist failed to drawn a distinction between those fractures produced by direct violence and those produced by muscular effort. It is well known that osseous union is practically unknown in those injuries which are produced by muscular action. This field of surgery was illumined by the work of Macewen, who in 1883 called attention to the fact that in fractures caused by muscular action, the posterior thick and elastic covering of the patella stretches before it gives way; that these fibrous bands are brought into apposition when the fracture is reduced, causing a heterogenous new tissue formation at the site of fracture that is, fibrous tissue between the two bony surfaces. In such cases the main consideration is not so much the suturing or the method by which the fragments are approximated as it is the removal of this incarcerated fibrous material from between the fractured ends which is interfering with bony union. To become dogmatic and say that every such case should be oper. ated upon, is going too far, as is shown by the course of the eminent authorities now in charge of the case of the Prince of Wales. He is under the care of Macewen, who practically originated suturing the patella, but is being treated by the older methods. There are certain conditions of the body of the royal patient which contraindicate his being treated along the operative line.

In fractures produced by direct violence there is comminu tion, but no stretching or incarceration of fibrous tissue, and in such cases we may reasonably expect firm bony union from any method which will bring the surfaces of bones into apposition. Intervening fibrous tissue must be removed if you seek bony union, and incarceration of tissue always occurs where the injury is the result of muscular action, which, of course, constitutes the great majority of such injuries. A body which would become authoritative in its field could hardly commit itself to the proposition that every case of patellar fracture should be operated upon, and to that extent I take exception to the paper.

Dr. W. J. MAYo of Rochester, Minn.-.I was very much pleased and interested in Dr. Jay's paper. Dr. Hodges struck the keynote when he said that in those cases of fracture of the patella caused by indirect violence the difficulty in securing union is from the interposition of fibrous tissue between the broken fragments. Macewen gave us the reason why in some cases of fracture of the ratella we had considerable separation of the fragments and still good functional use of the joint se. cured. It is because that portion of the anterior ligament, of which the patella is but a part, is separated, while the lateral xpansion of the tendon holds the joint in good position,

Another point to be considered is the best time for operation. The knee-joint is a good deal more dangerous than the peritoneal cavity, the reason for which is its liability to infection. In addition to that, it has not nearly the power of protecting itself that the peritoneal cavity has; it has not the lymphatic eupply. If we get infection of the knee-joint, we may not only lose the joint but the patient's life. A slight infection of the peritoneal cavity would be harmless as compared with a simiar infection of the knee-joint, for the reason that nature has not prepared that protection by which she provides a certain immunity from infection and traumatism in the peritoneal cavity. As to the time for operation, we will be governed argely by the condition of the skin. If the skin is not bruised we can operate at any time, but if it is bruised or covered with blisters, on account of the liability of carrying infection into the joint, surgical intervention should be delayed until a more favorable opportunity presents itself. In connection with operation, I do not think the finger should be allowed to touch the wound. Soiled fingers, which can not be avoided in the peritoneal cavity and are practically innocuous under ordinary circumstances, in the knee joint prove dangerous. The method of working entirely with instruments is good, sound surgical practice.

Dr. JAY (closing the discussion)-I do not know that I have anything in particular to say in my closing remarks. Probably most of you consider the paper a little radical. However, as I said in my paper, when there is a fracture of the patella, with pain in the joint, effusion of blood and synovial fluid in it ecchymosis, etc., I have no hesitation in opening into the joint, cleaning out the débris, stitching the fragments together, believing that I will have a good result. I call it good surgery to do this. If the lower end of the patella is broken off, does not penetrate the joint, and there is no effusion of blood or of synovial fluid into the joint, there is no necessity for opening the joint. It is not good practice to let a knee-joint filled with extravasated fluid or blood with fibrous tissue incarcerated between the fragments go unoperated. By external applications you can not get the fragments together, nor would you get bony union in such a case if the fragments could be brought into apposition. The interposition of fibrous tissue or of other material between the broken bone certainly prevents you from bringing the fracture in apposition.

Dr. Grant - What would you do in a case where there was no interposition of material between the fragments and there was simply eff used blood?

Dr. JAY-.-You do not wait for the interposition of fibrous tissue. I have operated on fracture of the patella for the last fifteen years, and I have never had an unfavorable result. Before I had carried out the treatment I have described I had the fragments one and two inches apart, particularly in the cases of old men, and they needed a support all the time. I do not operate on every fracture of the patella where the knee joint is opened, the aponeurotic structures are torn, and there is an effusion of blood. I have had three cases in the last six months in which the fractures were caused by external violence. These were railroad cases. In one case in particular, the fracture of the patella was caused by a railroad tie falling on the man's leg. I did not see him for five or six days after the injury, at which time the knee-joint measured twice what its fellow did. I could not tell definitely whether there was a fracture or not at that time. He was brought to the hospital hot antiseptic fomentations were applied to reduce the swelling, and then an incision was made, through which a pint of clotted blood was turned out of the knee-joint. The cavity of the joint was irrigated with boracic acid and bichlorid, the tissues washed and rewashed until they were thoroughly cleansed, and the patella sutured. This treatment was followed by a excellent result in this case.

\section{THE BACILLUS AEROPHILUS.}

\section{OCCURRENCE IN CHRONIC SUPPURATIVE OTITIS MEDIA.}

Presented to the Section on Laryngology and Otology at the cinth Annual Meeting of the Anerican Medica

BY LEWIS S. SOMERS, M.D.

PHILADELPHIA, PA.

Zaufal $^{1}$ states that a pure culture of any microorganism is rarely found in the secretion of otitis media purulenta chronica, and as a rule, there is a variety of bacteria, particularly in neglected otorrhea, 
where the secretion is stagnant. In acute otitis media suppurativa, pure cultures are found more frequently than when the disease has been neglected and assumes a chronic form; usually, however, various bacteria are associated, the most common being the diplococcus pneumonia, streptococcus pyogenes, staphyloccocus pyogenes albus and pyogenes aureus. More infrequently are found the staphylococcus tenuis, bacillus tenuis, micrococcus tetragenus and the bacillus pyocyaneus. That the micro-organisms obtained from the discharge in chronic suppurating otitis at its early stage are the cause of the morbid changes in the middle ear there can be no doubt, but in the form of the disease under consideration the bacteria found after the suppurating process has continued for a considerable time probably bear little or no relation to the primary disorder, secondary infection occurring through the perforation in the membrana tympani.

The following case, in which the bacillus aerophilus was found in pure culture, was discovered during the study of a series of cases of chronic suppurative otitis media, to ascertain the micro-organisms present in the discharges: W. C., aged 10 years, had a suppurating right middle ear since 1 year of age. No cause, such as the exanthemata, could be ascertained as producing the original otitis, and as the boy was a member of the lowest class of society, he presented every facility for secondary infection of the diseased ear. Examination showed the external auditory canal to be filled with foul pus, among which were shreds of gelatinous mucoid material, and after this was removed, the epidermal lining of the canal was seen to be macerated, with the epithelium proliferating and being discharged with the mucopurulent matter in the form of débris. Close to the tympanic ring was a group of bleeding granulations, surmounting a small particle of dead bone; there was a large ragged perforation in the posterior-inferior segment of the mem. brana tympani, through which a probe could be passed and which grated against necrosed bone in the tympanic cavity.

The pus discharged from the ear presented nothing differing in any way from that observed in long-continued cases of otitis media suppurativa, being of the usual yellowish.green color and possessing the foul odor peculiar to these cases. After the canal had been rendered as sterile as possible, a minute amount of pus was obtained on a platinum point, from deep in the middle ear, and from this a stab culture was made in a gelatin tube; a whitish growth developed in forty-eight hours, entire liquefaction taking place in seven days, with the development of the odor as observed in the patient's ear; the growths then sunk to the bottom of the tube, while the supernatant gelatin was liquefied and turbid. Otber cultures made on gelatin tubes, from the pus obtained at different times from the middle ear, presented the same biologic characteristics, and at no time during the examination did other micro-organisms become evident, either from the original culture or during the entire course of the investigations, which extended over several months.

On agar-agar smear cultures there was produced a creamy, yellow colored growth of waxy appearance; there was no liquefaction, and the entire surface of media was covered with the growth. Potato smear cultures showed a yellowish, irregular growth, waxy and dull looking, granular over the entire surface and with a slight odor. A series of gelatin plates were made after the Koch method, and the bacillus on these first appeared in colonies of minute yellow, round and pear-shaped granules, soon surrounded by a zone of liquefaction, which gradually increased in dimensions until the gelatin was entirely liquefied and clear on the surface, the colonies having gravitated to the bottom of the plates: Microscopic examinations were made of the original pus from the tym. panic cavity and of the varions colonies developed in the culture-media used at different times; carbolfuchsin was used as the principal stain in all examinations. From the pus obtained from the ear and original gelatin cultures, slides were prepared after the usual manner, and the bacilli were found in large numbers, to the exclusion of all other forms of germ life. It did not differ from the description given by Sternberg ${ }^{2}$, in which he figures it as slender rods of various lengths, about two-thirds as thick as the bacillus subtilis and frequently united in jointed filaments. At various stages in the life history of the bacillus, the size was smaller or larger, depending upon the duration of growth; in the early stages ovoid spores were present in large numbers, and a few hours later the bacilli would be seen as small rods, apparently immature; these would increase in size until the fully developed bacterium was seen.

The bacillus aerophilus is found, as far as has been studied, as an accidental contamination, probably from the air. It is an aerobic, liquefying, non-motile bacillus, with oval spores, and grows on the usual culture-media at the room temperature. It is impossible to ascertain the original micro-organism responsible for the primary infection of this case, but as a mere gratuitous supposition we may venture to sug. gest that several forms existed at various times during the course of the tympanic suppuration, and at the time the cultures were made the bacillus aerophi. lus had displaced the other forms of germ life and existed in the tympanic cavity as an individual growth. As a usual rule in the chronic cases, investigators have found a greater number of bacilli than cocci, staphylococci being almost constantly present.

Lermoyez and Helme ${ }^{3}$ make the following observations in regard to the infective agencies concerned in otitis media purulenta: 1. Acute otitis media is most often of microbic origin; the pus, obtained first from paracentesis of the drum membrane, generally contains but a single pathogenic species. 2. The streptococcus and the pneumococcus are the microbes usually found; the staphylococcus is rarely found at this period, and is generally found with other species. 3. After a varying period, a secondary infection is often grafted on the primary infection and takes the place of the latter. 4. This secondary infection is the result of the staphylococcus, especially of the white variety. 5. It determines the transition of the purulent otitis media from the acute to the chronic stage. Secondary infection of the tympanic cavity determining the chronic phase of the morbid process may occur either through the Eustachian tube from the nasopharyns, or from the external auditory canal and the perforation in the membrana tympani.

Pez and Gradenigo ${ }^{4}$ conclude that general secondary infection of the middle ear is of nasopharyngeal origin and very rarely does the secondary infection come from the external auditory canal through the perforation in the tympanic membrane. This somewhat comprehensive statement made by these observers is true in many cases, but it is frequently found that secondary infection, as in the case here described, 
results through the external canal; and usually the acute stage of an inflammation, one of their most im. infecting material is carried to the tympanic cavity portant reasons for denouncing these being the liabil. by neglect of aseptic precautions in the treatment of ity of favoring the development of granulations and of the previous acute otitis.

BIBLIOGRAPHY.

1 Zaufal:- Quoted by L. Stern, Metz. Archives of Otology, April, 1896. 2 Sternberg: Manual of Bacteriology.

3 Lermoyez and Helme: Annales des mal. de l'oreille et du larynx January, 1895.

4 Pez and Gradenigo: Ibid. July, 1895 .

3554 North Broad Street.

SUPPURATIVE DISEASE OF THE EAR.

THE PRESENCE OF POLYPI AND GRANULATIONS THEREIN NOT AN UNFAYORABLE INDICATION.

Presented to the Section on Laryngology and Otology, at the Fortyninth Annual Meeting of the American Medical Association, held at Denver, Colo., June 7-10, 1898.

BY LOUIS J. LAUTENBACH, M.D.

PHILADELPHIA, PA.

Some years ago, after having considerable experience followed by rather favorable results with cases of suppurative disease of the middle ear, complicated with granulations and polypi, I reported ${ }^{i}$ a few of my cases and drew therefrom the conclusion that "restoration of function in these cases is more frequent as well as more thorough than in chronic suppurative cases of equal duration without growths or polypi. I do not mean to say that this is universally so, but that it is usually the case." When this was written I intended it to apply to benignant growths, not to malignant or specific tumors. After several more years of work of this kind, I find my former opinions concerning these growths but confirmed and strengthened by the additional experience. In this paper I shall speak only of such growths as granulations, granulation tumors, the angiofibromata or mucous papillomata, the fibromata and the myxomata, and do not include such growths as the sarcomata, epithelioma, tubercle, lupus, etc. In the former paper I considered this exclusion as being understood, these malignant growths being very rare, much less than 1 per cent. being of this nature. Again, as designated in my original title, I speak of growths in the middle ear only, not pretending to speak of such as occur from the surface of the drum. head or external thereto.

Of these benign growths, by far the greater number are granulations and granulation tumors, these amount. ing to more than half the entire total of ear tumors. The next in frequency are the mucous papillomata or the angiofibromata. This is the variety present in about two-thirds of the remaining cases, while the fibromata comprise about all the balance except some 1 or 2 per cent. of the myxomatous variety of polyp. That the far greater number, in fact, possibly all, of these growths are the direct result of inflammatory action is apparent when we consider the conditions under which they develop; for all practical purposes never being present in the middle ear unless there has been an anterior inflammation of the drumhead, with congestion, exudation with suppuration and perforation.

The conditions under which the new growths appear are congestion or inflammation, with the presence of moisture. The latter essential is evidently considered of great moment by the large class of surgeons of today who denounce the use of heated cloths, and especially of hot poultices on the ear during the

1 Lautenbach, Louis J.: Improvement of Hearing after the Removal f Polypi and Granulations from the Middle Ear. Proceedings of the Pennsylvania State Medical Society, 1893. polypi. This moist inflammation or suppuration of the middle ear can further develop in various ways: 1. The inflammation. may gradually subside, the exudation lessening, the enlarged and leaking vessels gradually shrinking to a more nearly normal caliber, the diseased tissues proliferating where there has been destruction, and being absorbed where there has been thickening and exudation, and if the process has not been too severe, no fibrous or connective tissue bands having been produced within the ear, the structure as well as the function may be restored. 2. The process may have contined to the stage of organization of the elements of inflammation, with the formation of fibrous or cicatricial bands; and with the cessation of the inflammation and the closure of the perforation, we have healing, with impairment of structure and limitation of function. 3. We may have complete subsidence of the inflammation, with the formation of connective and perhaps fibrous tissues, with a cicatricial healing of the edges of the opening of the drumhead, and with considerable impairment of the structure and function. 4. We may have subsidence attended by necrotic processes, involving the soft tissues as well as the kone, eventuating in great impairment to the ear. 5. We may have it subside only after a most virulent and extensive inflammation, destroying through its violence important ear structures, and perhaps some of the surrounding tissues. Here, of course, destruction of structure is accompanied by destruction of function, 6. We may have the inflammation as it continues constantly producing increased sclerosis or increased necrosis, the amount of the impairment being proportioned to the length and variety of the inflammatory processes. $7 . \mathrm{We}_{\mathrm{e}}$ may have the inflammation continue; and as it continues, hyperplastic processes being at work, new structures appear, giving rise to granulations and granulation tumors, if due to the organization of the inflammatory exudate, and the other forms of benign polypi. when the inflummatory action modifies the normal furction of the epithelial and fibrous tissues, stimulating them to a new development of their own tissues in varying forms. This varying development is probably more dependent upon the degree of vascularity present than upon any other factor.

I look upon these new growths as an evidence of an activity of the inflammatory action in a benign man. ner-not in the way of destruction or of necrosis, not in the way of a general fibrous thickening, not a sclerotic or hardening action, nor yet akin to the process of cicatrization-but rather an effort of nature to extend her benignant healing processes to as great an extent as possible, so that, although the healing will not be a primary one, it will yet be akin to that observed in other parts of the body - the healing by the formation of proud flesh. It allows the retention of the necessary vascularity, the nerve innervation remaining, until such time as, through artificial or natural means, everything is ready for a quick healing, and all we need is to take advantage of nature's open door. Through the presence of this prolonged increase of vascularity and of nerve energy, the various ear structures, the lining membrane of the middle air, the articulations, especially the stapedio temporal, as well as the ear nerve, are better preserved in structure and in function than they would be otherwise. This evi- 